\title{
miR-34c-5p targets Notch1 and suppresses the metastasis and invasion of cervical cancer
}

\author{
HUALI WEI $^{1}$, XIAOLAN WANG ${ }^{1}$, XIUMIN NIU $^{1}$, RUILI JIAO ${ }^{2}$, XIAOJUAN LI $^{3}$ and SUMEI WANG ${ }^{4}$ \\ ${ }^{1}$ Department of Gynecology and Obstetrics, Emergency General Hospital, Beijing 100028; \\ ${ }^{2}$ Department of Gynecology and Obstetrics, Chaoyang District Maternal and Child Health Hospital, Beijing 100020; \\ ${ }^{3}$ Department of Medical Records and Statistics, Emergency General Hospital, Beijing 100028; \\ ${ }^{4}$ Department of Gynecology and Obstetrics, Beijing Chaoyang Hospital, Capital Medical University, Beijing 100020, P.R. China
}

Received April 2, 2020; Accepted October 16, 2020

DOI: $10.3892 / \mathrm{mmr} .2020 .11759$

\begin{abstract}
Micro (mi)RNAs are crucial participants in the progression of cervical cancer (CC). Growing evidence indicates that miRNA (miR)-34c-5p is a pivotal tumor suppressor in numerous types of cancer and its functions in $\mathrm{CC}$ require further investigating. The present study demonstrated that there was a decreased level of miR-34c-5p in CC-associated cell lines compared with healthy control samples. It also demonstrated that miR-34c-5p targeted Notch1 and suppressed $\mathrm{CC}$ progression. Dual-Luciferase reporter assays verified the targeted relationship of miR-34c-5p and Notch1. The expression of Notch1 in HeLa cells was markedly reduced following miR-34c-5p overexpression and the proliferation, migration and invasion of HeLa cells were reduced although apoptosis was accelerated. However, overexpression of miR-34c-5p was reversed following the addition of Notch1, which supported the finding of the targeted relationship between miR-34c-5p and Notch1. Flow cytometry demonstrated that miR-34c-5p inhibited the proliferation of HeLa cells while accelerating apoptosis. The present study concluded that miR-34c-5p was a tumor suppressor in $\mathrm{CC}$ and may be a novel measure for the future treatment of $\mathrm{CC}$.
\end{abstract}

\section{Introduction}

Cervical cancer (CC) is known as one of the most common types of gynecological cancers worldwide and it is also the fourth leading cause of female mortality (1). Advances in early diagnosis, surgical resection and chemotherapy/radiation enable patients to receive effective treatments, however, $\mathrm{CC}$ prognosis remains poor (2). Causes of death in patients

Correspondence to: Dr Sumei Wang, Department of Gynecology and Obstetrics, Beijing Chaoyang Hospital, Capital Medical University, 8 Workers Stadium South Road, Chaoyang, Beijing 100020, P.R. China

E-mail: sumeiwang88@163.com

Key words: cervical cancer, microRNA-34c-5p, Notch homolog 1, proliferation, migration and invasion with $\mathrm{CC}$ are mainly cancer progression, metastasis and resistance (3). Therefore, investigations into the mechanism and progression of tumorigenesis may provide novel insights for the development of new treatment methods of CC.

MicroRNAs (miRNAs or miRs) are 22nt small non-coding RNA molecules, which are closely implicated in gene expression (4). miRNAs either degrade certain specific genes or inhibit their expression by binding to 3'-untranslated regions (3'-UTRs) of their mRNAs in a complimentary manner $(5,6)$. miRNAs are widely involved in various biological processes including proliferation, tumor metastasis and drug tolerance $(7,8)$. miR-34c-5p has been reported to function as a tumor suppressor in numerous cancer types (9). However, whether the same miRNA serves as an oncogene or not is ultimately dependent on the characteristics of the target genes (10-12). The present study aimed at investigating the effects and mechanism of miR-34c-5p in CC.

The Notch signaling pathway is an evolutionarily conserved signaling pathway that mediates proliferation and differentiation as well as the survival and apoptosis of cells (13). The Notch signaling pathway encompasses Notch transmembrane receptors (Notch1-4) and their ligands (Delta-like 1, 3 and 4 and Jagged 1 and 2) (14). The Notch receptor features a single channel transmembrane protein and consists of an extracellular domain, a transmembrane domain and an intracellular domain. Once the Notch signaling pathway is activated by the ligand-receptor of joint cells, the Notch1 receptor is cleaved by $\gamma$-secretase, thereby releasing Notch1 intracellular domain (NICD) from the plasma membrane (15). Subsequently, NICD translocates into the nucleus and then participates in the transcription of other transcription factors to further regulate its downstream genes including members of Hes and Hey families (16). The Notch signaling pathway regulates the growth of numerous tissues and cells in a cellular context-dependent way, which further affects cell specialization, proliferation and apoptosis (16). A dysregulated Notch signaling pathway has been revealed in various types of cancer including CC (17). However, the interaction between miR-34c-5p and Notch1 remains to be elucidated. The present study was designed to explore the biological functions and mechanism of miR-34c-5p on $\mathrm{CC}$ at a molecular level, hoping to provide a novel approach to the current treatment of this disease. 


\section{Materials and methods}

Tissue collection. CC and adjacent tissues were collected from 30 patients aged between 29 and 72 who underwent surgical resections at the Emergency General Hospital (Beijing, China) between December 2017 and December 2019. One of the patients who received anticancer treatment was excluded from the study. All tissues were frozen by liquid nitrogen immediately and stored at $-80^{\circ} \mathrm{C}$ for later use. The experimental protocol was authorized by the Ethics Committee of Emergency General Hospital (approval no. 2017SY1503). Prior written informed consent was obtained from each patient.

Hematoxylin and eosin (H\&E) staining. Tissue samples were fixed in $4 \%$ formalin for $48 \mathrm{~h}$ at room temperature. The tissues were then paraffin-embedded, then cut to $5-\mu \mathrm{m}$-thick sections. Slides were subjected to H\&E stain according the manufacturer's instructions (Beyotime Institute of Biotechnology).

EdU analysis. An EdU detection kit (cat. no. C10310; Guangzhou RiboBio Co., Ltd.) was used to detect cell proliferation. Briefly, cells were plated in 24-well plates at a density of $5 \times 10^{4}$ cells/well and treated with $50 \mu \mathrm{M}$ EdU solution for $4 \mathrm{~h}$ at $37^{\circ} \mathrm{C}$. The cells were then fixed with $4 \%$ paraformaldehyde for $10 \mathrm{~min}$ at room temperature and treated with $0.5 \%$ Triton X-100 at room temperature for $5 \mathrm{~min}$. The nuclei were labeled with DAPI (Guangzhou RiboBio Co., Ltd.).

Cell culture and transfection. Human CC cell lines (C33A, CaSki, HeLa and $\mathrm{SiHa}$ ) and human immortalized normal cervical cell line (Ect1/E6E7) were acquired from the American Type Culture Collection (ATCC). 293T cells were purchased from the Cell Bank of Type Culture Collection of the Chinese Academy of Sciences. All cell lines were cultured in DMEM (HyClone; Cytiva) supplemented with 10\% FBS (HyClone; Cytiva), $100 \mathrm{U} / \mathrm{ml}$ penicillin and $100 \mu \mathrm{g} / \mathrm{ml}$ streptomycin (Beyotime Institute of Biotechnology) in a humidified incubator containing $5 \% \mathrm{CO}_{2}$ at $37^{\circ} \mathrm{C}$. The miR-34c-5p mimics (5'-AGGCAGUGUAGUUAGCUGAUUGC-3'), miR-34c-5p mimics negative control (5'-ACUACUGAGUGACAGUAG A-3'), miR-34c-5p inhibitors (5'-GCAAUCAGCUAACUA CACUGCCU-3') and miR-34c-5p inhibitors negative control (5'-UUCUCCGAACGUGUCACGUTT-3') were synthesized by Guangzhou RiboBio, Co., Ltd. Full-length Notch1 from human cDNA library was inserted a pcDNA3.1 vector (Invitrogen; Thermo Fisher Scientific, Inc.). A pcDNA3.1 vector alone (empty plasmid) served as a negative control. Cells were transfected using Lipofectamine ${ }^{\circledR}$ LTX reagent (Invitrogen; Thermo Fisher Scientific, Inc.) according to the manufacturer's instructions. HeLa cells were transfected either with miR-NC (50 $\mathrm{nM})$ or miR-34c-5p mimics (50 $\mathrm{nM})$ and/or pcDNA3.1/Notch1 vector (100 nM) or pcDNA3.1 (100 nM). In addition, CaSki cells were transfected with anti-NC (50 nM) or miR-34c-5p inhibitors (50 nM). Following transfection for $48 \mathrm{~h}$, cells were harvested for subsequent experiments and repeated 4 times.

Cell Counting Kit-8 (CCK-8) assay. HeLa cell viability was detected by CCK- 8 assay (Beyotime Institute of Biotechnology). HeLa cells $\left(1 \times 10^{3}\right.$ cells/well) were cultured in 96-well plates for 0, 24, 48 and $72 \mathrm{~h}$, four times in each time group. At a fixed time, $10 \mu \mathrm{l}$ of CCK- 8 was added into each well and incubated for $3 \mathrm{~h}$. The optical density and $450 \mathrm{~nm}$ of each well was determined in quadruplicate using Multiskan MK3 (Thermo Fisher Scientific, Inc.).

Transwell assay. Migration and invasion abilities of cells were determined through Corning Transwell chambers (Corning, Inc.). For the detection of invasion capability, an $8-\mu \mathrm{m}$ pore size Transwell membrane filter was precoated with $30 \mu 1$ Matrigel $^{\mathrm{TM}}$ (BD Biosciences) at $37^{\circ} \mathrm{C}$ for $4 \mathrm{~h}$. In the migration and invasion detection, HeLa cells $\left(5 \times 10^{4}\right.$ cells) were resuspended in $100 \mu \mathrm{l}$ DMEM without the addition of FBS before being transferred to the upper chamber. A total of $600 \mu 1$ of DMEM supplemented with $10 \%$ FBS was added to the lower chamber. Cells were incubated for $12 \mathrm{~h}$ before being fixed with $4 \%$ paraformaldehyde for $10 \mathrm{~min}$ at room temperature and stained with $0.5 \%$ crystal violet at room temperature for $20 \mathrm{~min}$. The number of stained cells randomly selected from six fields were counted with images captured under a light microscope (Olympus Corporation; magnification, x100) and repeated four times.

Western blotting. Tissue samples and treated cells were lysed by radioimmunoprecipitation assay buffer (Beyotime Institute of Biotechnology). Protein concentrations were determined by BCA Protein Assay kit (Pierce; Thermo Fisher Scientific, Inc.). A quantity of protein extract (30 $\mu \mathrm{g}$ total protein/lane) was resolved via $10 \%$ SDS-PAGE and transferred onto PVDF membranes and blocked with 5\% dried skimmed milk at room temperature for $1 \mathrm{~h}$. The PVDF membranes were incubated with primary antibody (Notch1; dilution 1:500; cat. no. ab52627; Abcam) and $\beta$-actin antibody (dilution 1:1,000; cat. no. ab8227; Abcam) with gentle agitation at $4^{\circ} \mathrm{C}$ overnight and then treated with secondary antibody (horseradish peroxidase-labeled goat anti-rabbit; dilution 1:1,000; cat. no. ab150077; Abcam) at room temperature for $2 \mathrm{~h}$. $\beta$-actin served as a loading control. Protein bands were visualized via an enhanced chemiluminescence system (Beyotime Institute of Biotechnology) and repeated four times. Western blots were quantified by Image J software (V 1.46; National Institutes of Health).

$R N A$ extraction and reverse transcription-quantitative (RT- $q)$ $P C R$. A total of $2 \mu \mathrm{l}$ RNA (at a concentration of $200 \mathrm{ng} / \mu \mathrm{l}$ ) was extracted from $2 \times 10^{6}$ cells and tissues with TRIzol ${ }^{\circledR}$ reagent (Thermo Fisher Scientific, Inc.). cDNA was synthesized by $\operatorname{TaqMan}^{\circledR}$ MicroRNA Reverse Transcription kit (Thermo Fisher Scientific, Inc.) according to the manufacturer's protocols. To quantify miRNA and mRNA, a qPCR assay was performed with $\mathrm{iQ}^{\mathrm{TM}}$ SYBR ${ }^{\mathrm{T}}$ Green Supermix (Bio-Rad Laboratories, Inc.) on the platform of an $\mathrm{iCycler}_{\mathrm{iQ}} \mathrm{Q}^{\mathrm{TM}} \mathrm{qPCR}$ detection system (Bio-Rad Laboratories, Inc.). Relative levels of miR-34c-5p and Notch1 were calculated as an inverse $\log$ of $2-\Delta \Delta \mathrm{Cq}$ and normalized to the reference gene (18), repeated four times. Conditions of the thermocycling were as follows: $95^{\circ} \mathrm{C}$ for $10 \mathrm{~min}$; followed by 40 cycles at $95^{\circ} \mathrm{C}$ for $15 \mathrm{sec}$ and $60^{\circ} \mathrm{C}$ for $1 \mathrm{~min}$; annealed at $55^{\circ} \mathrm{C}$ for $30 \mathrm{sec}$; and elongated at $72^{\circ} \mathrm{C}$ for $3 \mathrm{~min}$. $\beta$-actin was considered as an internal reference and employed to analyze the expression 
of Notch 1 gene. U6 was regarded as an internal control for the detection of miR-34c-5p expression. Primers were as follows: Notch1-forward (F), 5'-GAGGCGTGGCAGACT ATGC-3' and Notch1-reverse (R), 5'-CTTGTACTCCGTCAG CGTGA-3'; miR-34c-5p RT primer, 5'-GTTGGCTCTGGT GCAGGGTCCGAGGTATTCGCACCAGAGCCAACGCAA TC; miR-34c-5p-F, 5'-CGGAGGCAGTGTAGTTAGCT-3' and miR-34c-5p-R, 5'-GTGCAGGGTCCGAGGT-3'; U6 RT primer, 5'-AACGCTTCACGAATTTGCGT-3'; U6-F, 5'-CTC GCTTCGGCAGCACA-3' and U6-R, 5'-AACGCTTCACGA ATTTGCGT-3'; $\beta$-actin-F, 5'-CATGTACGTTGCTATCCA GGC-3' and $\beta$-actin-R, 5'-CTCCTTAATGTCACGCAC GAT-3'.

Flow cytometry. According to the manufacturer's protocol, estimation of the apoptosis rate was performed with Annexin V-PI detection kit (Beyotime Biotechnology Institute). Cell cycles, proliferation and apoptosis rate of each sample were analyzed by flow cytometry (Cytomics Fc500 MPL Flow Cytometer; Beckman Coulter, Inc.) and repeated four times. All data were analyzed with ModFit LT 3.0 (Verity Software House, Inc.).

Luciferase reporter assay. Wild-type (WT) or mutant (MUT) Notch1-3'UTR with the miR-34c-5p binding site was loaded into psicheck 2 vector (Promega Corporation). The $293 \mathrm{~T}$ cells $\left(1 \times 10^{5}\right.$ cells/well $)$ were co-transfected with $0.1 \mathrm{mg}$ psiCHECK2-WT Notch1-3'-UTR or $0.1 \mathrm{mg}$ psiCHECK2-MUT Notch1-3'-UTR and $10 \mathrm{nM}$ miR-34c-5p mimics or $10 \mathrm{nM}$ miR-34c-5p inhibitors using Lipofectamine ${ }^{\circledR}$ 2000 (Invitrogen; Thermo Fisher Scientific, Inc.) according to the manufacturer's protocol. Cells were cultured at $37^{\circ} \mathrm{C}$ for $48 \mathrm{~h}$ and then analyzed for luciferase activity according to the manufacturer's protocol (GeneCopoeia, Inc.) and the experiment was repeated four times. Luciferase activity was standardized by comparison with Renilla luciferase activity.

Bioinformatics prediction. Potential target genes of miR-34c-5p were identified by using the online prediction system TargetScan 7.1 (http://www.targetscan.org).

Statistical analysis. Data were presented as the mean \pm standard error of the mean. SPSS 13.0 software (SPSS, Inc.) was used for statistical processing. Unpaired Student's t-test or one-way ANOVA was used for data analysis. Assays of significant difference were subjected to Tukey's post hoc test. $\mathrm{P}<0.05$ was considered to indicate a statistically significant difference.

\section{Results}

miR-34c-5p expression is downregulated in human CC tissues and related cells. The morphology of $\mathrm{CC}$ and adjacent tissues (control group) was observed using H\&E staining (Fig. S1). To verify the role of miR-34c-5p in CC, miR-34c-5p expression was initially determined in 30 pairs of $\mathrm{CC}$ tissues and adjacent tissues by RT-qPCR, which indicated that the miR-34c-5p expression was significantly reduced in tumor tissues compared with normal tissues (Fig. 1A). The level of miR-34c-5p expression was detected in four types of CC cells (C33A, SiHa, HeLa and CaSki) by RT-qPCR and the results indicated that the expression of miR-34c-5p was significantly
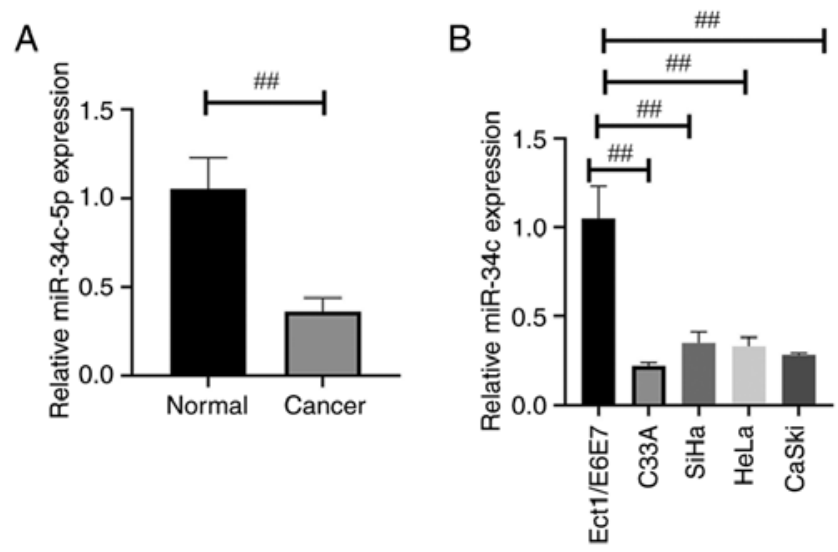

Figure 1. miR-34c-5p expression is downregulated in human cervical carcinoma tissues and cells. (A) RT-qPCR assay was used to detect the miR-34c-5p expression in 30 pairs of human cervical carcinoma tissues and control. (B) The miR-34c-5p expression in cervical carcinoma cell lines and normal cervical cell line were determined via RT-qPCR. ${ }^{\# \prime} \mathrm{P}<0.01$. miR, microRNA; RT-qPCR, reverse transcription-quantitative PCR.

decreased in $\mathrm{CC}$ cell lines when compared with that in Ect1/E6E7 cells (Fig. 1B).

miR-34c-5p inhibits CC cell proliferation and enhances cell apoptosis. miR-34c-5p was overexpressed or silenced in HeLa cells to ascertain its role in the development of CC (Fig. 2A). CCK-8 (Fig. 2B) and EdU analysis (Fig. 2C-D) indicated that the proliferation of HeLa cells was inhibited in the miR-34c-5p-mimics group, while an increase was revealed in miR-34c-5p-inhibitor-transfected HeLa cells (Fig. 2B-D). Flow cytometry was employed to detect cell proliferation, cell cycle and apoptosis with results revealing that miR-34c-5p mimics inhibited cell proliferation and promoted cell apoptosis (Fig. 3).

miR-34c-5p inhibits the migration and invasion capacities of CC cells. Transwell assays demonstrated that the both the migration and invasion abilities in miR-34c-5p-mimic-transfected HeLa cells were significantly reduced compared with the miR-NC group; however, that of miR-34c-5p-silenced HeLa cells exhibited an increase in migration and invasion abilities (Fig. 4).

Notchl acts as a target of $m i R-34 c-5 p$. The underlying mechanism of miR-34c-5p was investigated in CC progression, the potential targets of which were predicted using TargetScan. The results demonstrated that Notch1 mRNA 3'-UTR possesses highly conserved binding sites for miR-34c-5p to bind with (Fig. 5A). Correlations between Notch1 and miR-34c-5p were analyzed through luciferase reporter assay. Luciferase reporter plasmid containing wt/mut 3'-UTR human Notch1 binding site was co-transfected with miR-34c-5p mimics into the $293 \mathrm{~T}$ cells. miR-34c-5p mimics efficiently reduced the luciferase activity of Notch1 wt 3'-UTR in the 293T cells but no effects were observed in the cells transfected with the mut Notch1 3'-UTR (Fig. 5B). As it was identified as a specific target of miR-34c-5p (Fig. 3B), Notch1was highly expressed in CC (Fig. S2). The results of RT-qPCR and western blot analysis demonstrated that overexpression of miR-34c-5p disabled 

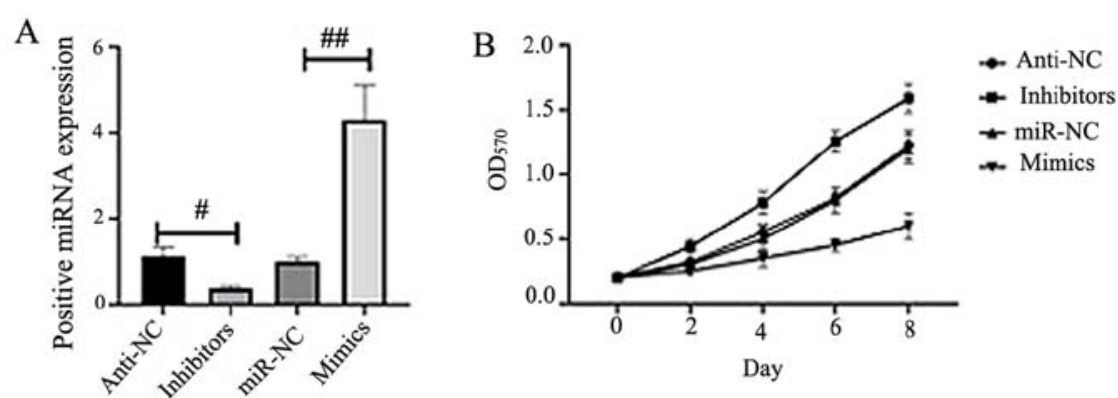

$\mathrm{C} \rightleftharpoons$

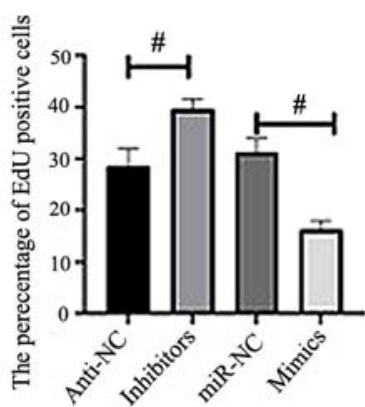

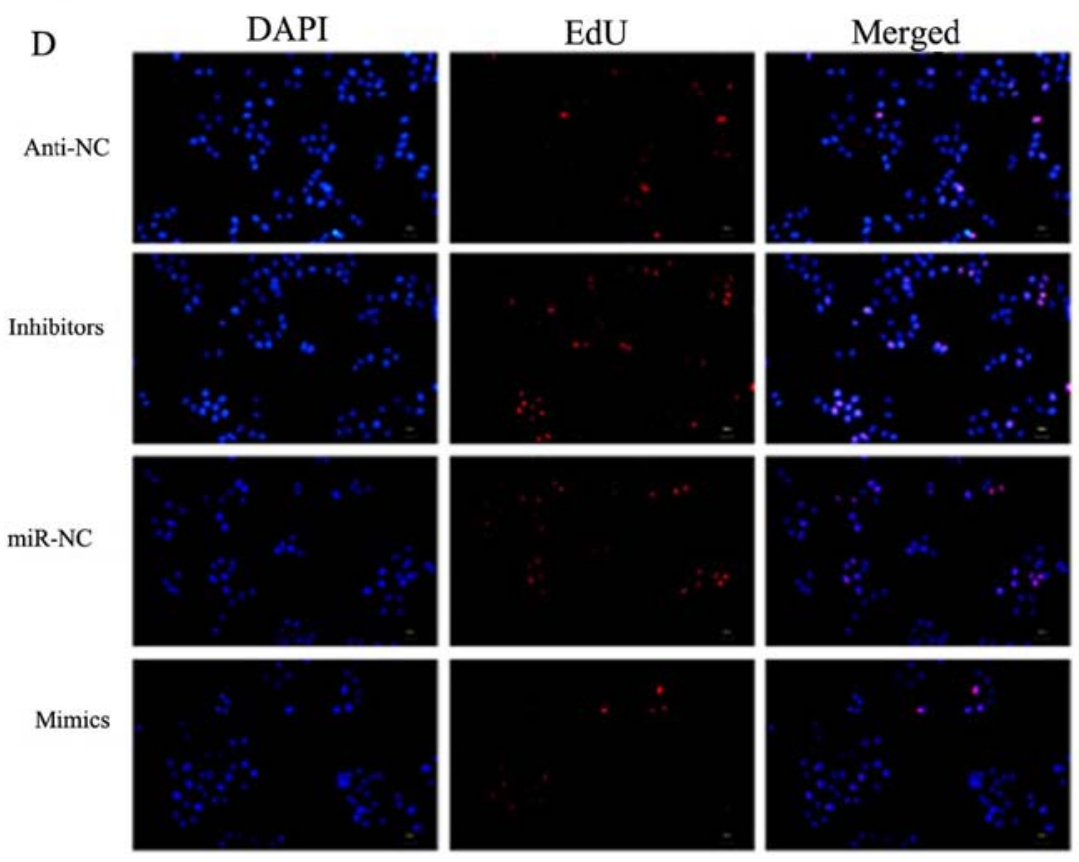

Figure 2. miR-34c-5p inhibits the capacities of cervical carcinoma cell proliferation. (A) miR-34c-5p expression levels in mimics/miR-NC and inhibitors/anti-NC transfected cells were determined by reverse transcription-quantitative PCR. (B) HeLa cell viability was assessed with a CCK-8 assay. (C and D) An EdU assay was used to analyze the proliferation rates of HeLa cells. Magnification, $\mathrm{x} 200 .{ }^{~} \mathrm{P}<0.05,{ }^{\# \prime} \mathrm{P}<0.01$. miR, microRNA; NC, negative control; OD, optical density.
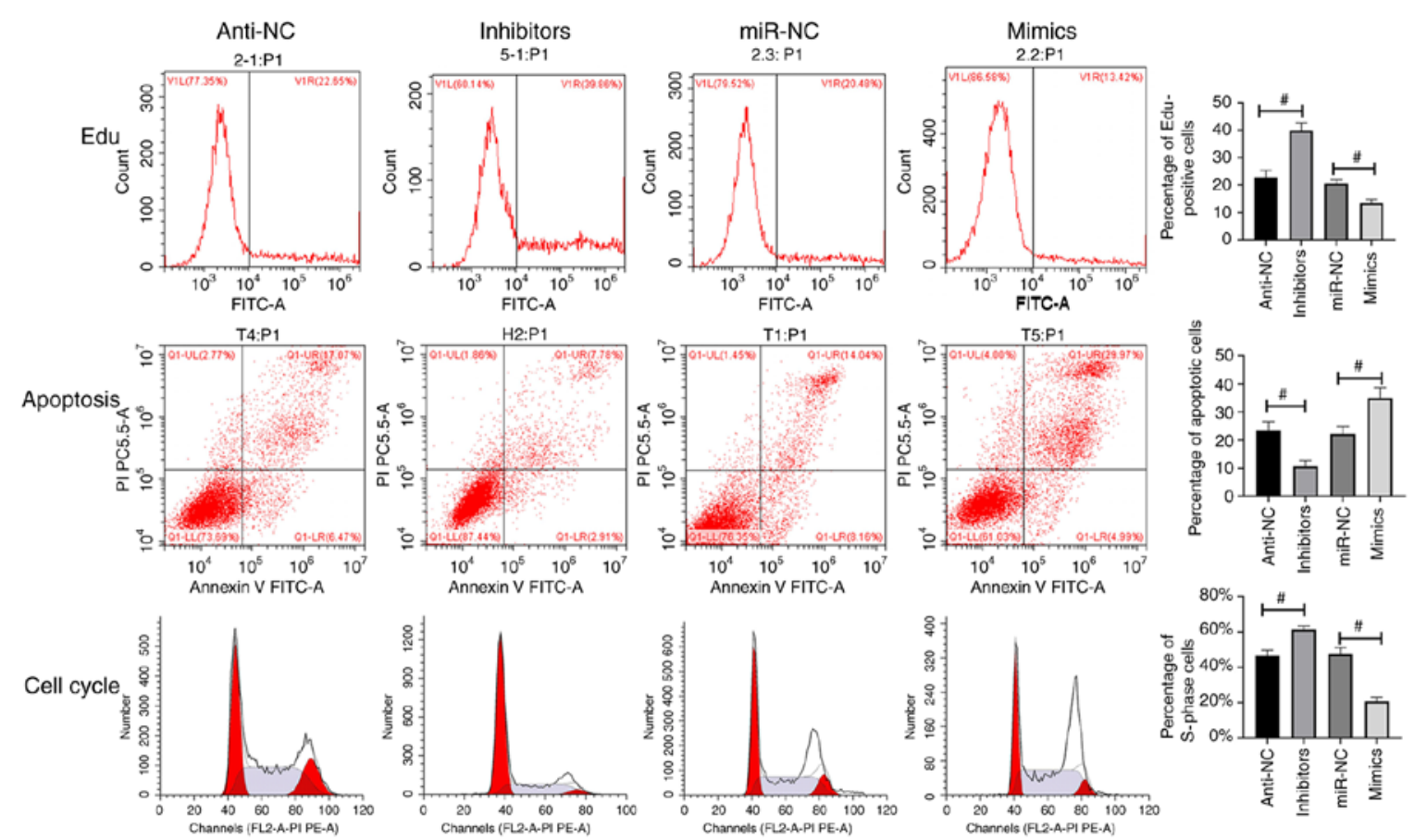

Figure 3. Cell proliferation, apoptosis and cell cycle were detected by flow cytometry. ${ }^{\#} \mathrm{P}<0.05$. NC, negative control; miR, microRNA. 
A

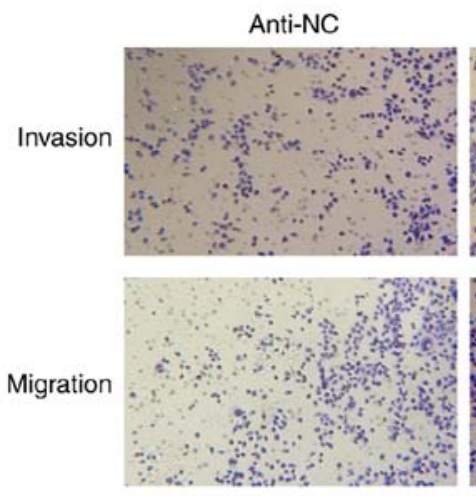

B

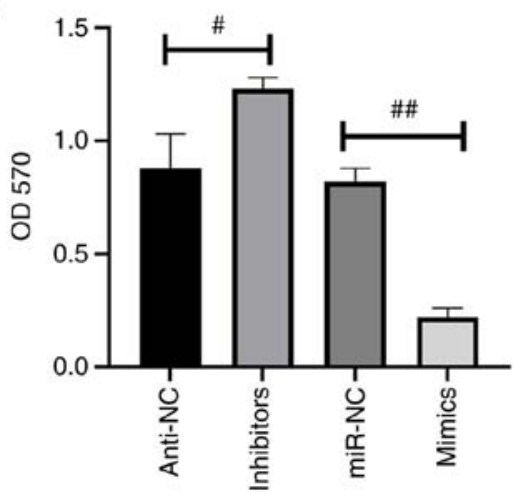

Inhibitors
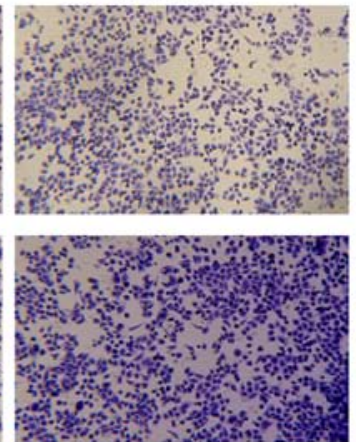

miR-NC

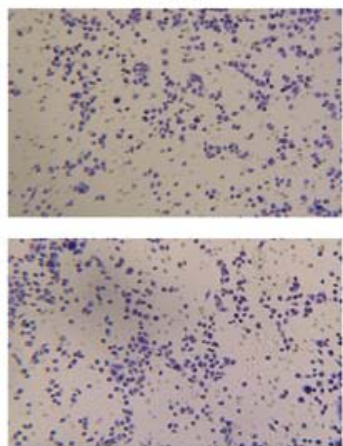

Mimics

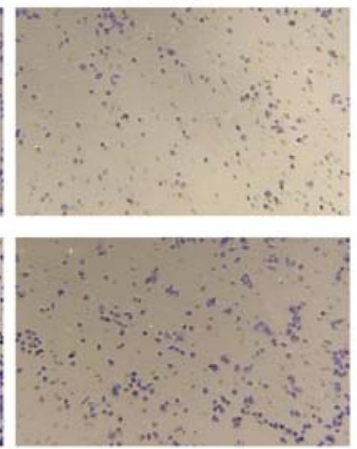

C

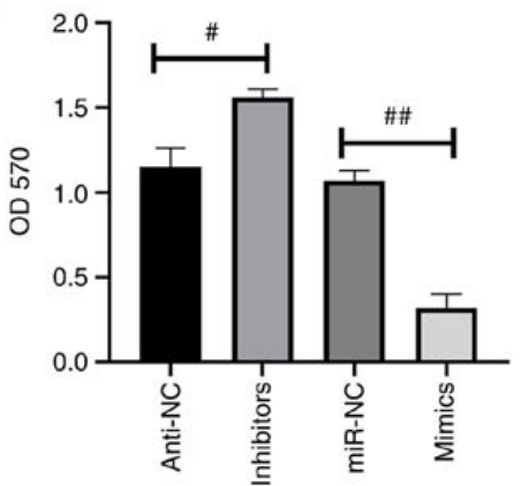

Figure 4. miR-34c-5p inhibits the capacities of cervical carcinoma cell migration and invasion. (A) Transwell assays were performed to analyze the migration and the invasion capabilities of HeLa cells. Magnification, $\mathrm{x} 200$. (B and C) Statistical results of the Transwell assays. ${ }^{\#} \mathrm{P}<0.05$, ${ }^{\# \#} \mathrm{P}<0.01$. NC, negative control; miR, microRNA; OD, optical density.

A

Position 180-186 of NOTCH1 3' UTR: 5' ...UAUUUUACACAGAAACACUGCCU...

||||||

hsa-miR-34c-5p: 3' CGUUAGUCGAUUGAUGUGACGGA

Position 180-186 of NOTCH1 3' UTR-MUT: 5' ...UAUUUUACACAGAAACACACGCU...

B

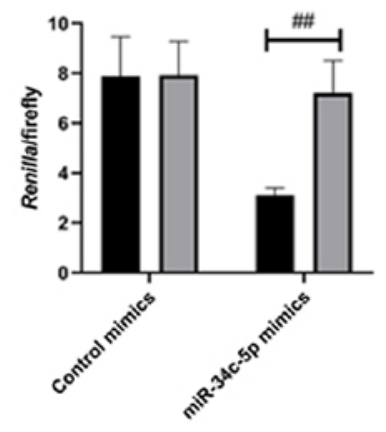

- Notch1-WT C 口 Notch1-MUT
D

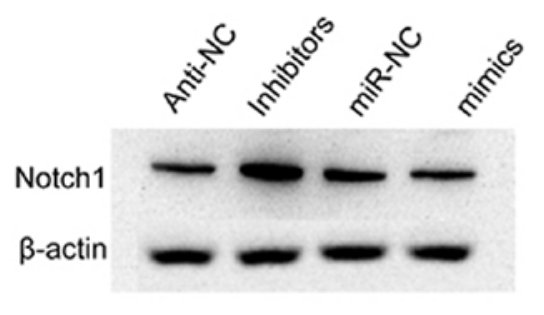

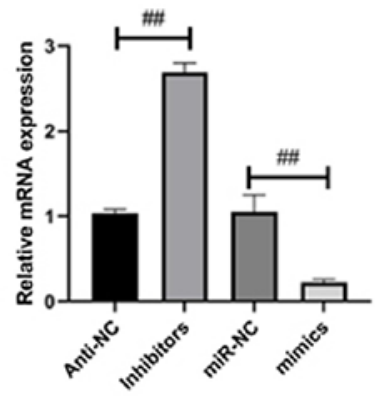

$E$

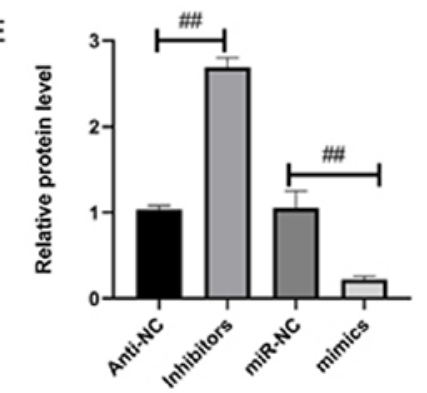

Figure 5. Notch1 is a target of miR-34c-5p. (A) The 3'-UTR of Notch1 mRNA encompasses a highly conserved binding site for miR-34c-5p matching. (B) 293T cells were co-transfected with miR-34c-5p mimics and WT Notch1 mRNA 3'-UTR/mut Notch1 mRNA 3'-UTR. Luciferase activity was analyzed after $24 \mathrm{~h}$ of transfection. (C) mRNA expression levels of Notch1 were determined in HeLa cells (D and E) The protein expression levels of Notch1 were detected in HeLa cells. ${ }^{\# \#} \mathrm{P}<0.01$. miR, microRNA; UTR, untranslated regions; WT, wild-type; hsa, Homo sapiens; MUT, mutant; NC, negative control. 
Notch1 expression in HeLa cells at mRNA and protein levels (Fig. 5C-E) in contrast to the miR-NC cells; the opposite results obtained from miR-34c-5p silencing in HeLa cells further demonstrated Notch 1 to be the target of miR-34c-5p.

\section{Discussion}

Previous studies have validated the relationship between cancer progression and dysregulated miRNA expression. Several miRNAs have been demonstrated to serve as either tumor suppressors or oncogenes in the development of CC (19-21). Despite numerous miRNAs acting as tumor suppressors and inhibitors of their target gene expression at a low level, they may also elicit an intense oncogene translation, thereby facilitating the development of tumors (22). Overexpressed oncogenic miRNAs may also result in an inhibitory effect on tumor suppressor genes (23).

miR-34c-5p has been revealed to be downregulated in types of cancer (10-12), although the mechanism in $\mathrm{CC}$ remains unclear. The present study revealed that the miR-34c-5p expression exhibited a significant decrease in CC and relevant cell lines, indicating that miR-34c-5p served an essential role in cell proliferation, metastasis and apoptosis of $\mathrm{CC}$. The aforementioned findings suggested that miR-34c-5p overexpression hindered the viability, proliferation, migration and invasion of different types of cancer cells and accelerated apoptosis in vitro.

miRNAs inhibit the expression of specific target genes, resulting in regulation of a variety of biological changes (24). miR-34c-5p is expressed at a low level in gliomas compared with normal brain tissues and normal glial cell lines (12). Overexpression of miR-34c-5p was revealed to inhibit U251 cell proliferation and result in S-phase arrest, $\mathrm{G} 0 / \mathrm{G} 1$ reduction and cell apoptosis (12). In gastric cancer, miR-34c-5p was revealed to directly bind to the $3^{\prime}$ UTR of the microtubule-associated protein tau (MAPT) and inhibit the expression of MAPT (25). The present study determined that miR-34c-5p directly targeted Notch1 mRNA and downregulated Notch1 expression, thereby inhibiting the progression of CC. Notch1 has also been reported to dissociate from the fused negative inhibitor in the primary cilium and then converts into an activated form and migrates to the nucleus $(26,27)$. Notch1 translocation enhances the expression of downstream target oncogenes including cyclin D1 and homeobox protein NANOG $(28,29)$. Notch1 has been revealed to be highly expressed as an oncogene in various cancers including non-small cell lung, breast, liver and gastric cancer (30-33). The present study confirmed that miR-34c-5p impaired Notch1 expression by directly binding to the 3'-UTR of Notch1 mRNA. It also demonstrated a negative correlation between the level of miR-34c-5p and Notch1 mRNA in CC. In addition, it was observed that the overexpression of Notch1 in CC hindered the effect of miR-34c-5p on cell survival and metastasis. All the aforementioned underpin the hypothesis that Notch1 is the direct target of miR-34c-5p.

In conclusion, the present study demonstrated that miR-34c-5p was significantly downregulated in human CC and related cell lines and that overexpressed miR-34c-5p accelerated cell apoptosis and inhibited proliferation, invasion and migration of $\mathrm{CC}$ cells. The present study elucidated the biological traits of miR-34c-5p and Notch1 so as to further study their correlation, which may provide novel approaches for the treatment of CC.

\section{Acknowledgements}

Not applicable.

\section{Funding}

No funding was received.

\section{Availability of data and materials}

The datasets used and/or analyzed during the current study are available from the corresponding author on reasonable request.

\section{Authors' contributions}

SW, HW and XW conceived and designed the experiments. HW, RJ, XW, XN and XL conducted all the experiments. SW, HW and XW wrote and revised the manuscript. All authors read and approved the final manuscript.

\section{Ethics approval and consent to participate}

The present study was approved by the Ethics Committee of Emergency General Hospital (Beijing, China). Prior written informed consent was obtained from each patient.

\section{Patient consent for publication}

Not applicable.

\section{Competing interests}

The authors declare that they have no competing interests.

\section{References}

1. Liu M, Wang Z, Liu Q, Zhu $\mathrm{H}$ and $\mathrm{Xu} \mathrm{N}$ : Expression of Micro-RNA-492 (MiR-492) in human cervical cancer cell lines is upregulated by transfection with wild-type P53, irradiation, and 5-fluorouracil treatment in vitro. Med Sci Monit 24: 7750-7758, 2018.

2. Song TT, Xu F and Wang W: Inhibiting ubiquitin conjugating enzyme E2 $\mathrm{N}$ by microRNA-590-3p reduced cell growth of cervical carcinoma. Kaohsiung J Med Sci 36: 501-507, 2020.

3. Wang T, Feng J and Zhang A: miR-584 inhibits cell proliferation, migration and invasion in vitro and enhances the sensitivity to cisplatin in human cervical cancer by negatively targeting GLI1. Exp Ther Med 19: 2059-2066, 2020.

4. Jones RA, Franks SE and Moorehead RA: Comparative mRNA and miRNA transcriptome analysis of a mouse model of IGFIR-driven lung cancer. PLoS One 13: e206948, 2018.

5. Chen Z, Zhang M, Qiao Y, Yang J and Yin Q: MicroRNA-1297 contributes to the progression of human cervical carcinoma through PTEN. Artif Cells Nanomed Biotechnol 46: 1120-1126, 2018.

6. Lu HJ, Jin PY, Tang Y, Fan SH, Zhang XF, Wang F, Wu DM, Lu J and Zheng YL: microRNA-136 inhibits proliferation and promotes apoptosis and radiosensitivity of cervical carcinoma through the NF- $\kappa$ B pathway by targeting E2F1. Life Sci 199: 167-178, 2018.

7. Yin Z and Ren W: MicroRNA-217 acts as a tumor suppressor and correlates with the chemoresistance of cervical carcinoma to cisplatin. Onco Targets Ther 12: 759-771, 2019. 
8. Yu LM, Wang WW, Qi R,Leng TG and Zhang XL: MicroRNA-224 inhibition prevents progression of cervical carcinoma by targeting PTX3. J Cell Biochem 119: 10278-10290, 2018.

9. Ding W, Xin J, Jiang L, Zhou Q, Wu T, Shi D, Lin B, Li L and Li J: Characterisation of peripheral blood mononuclear cell microRNA in hepatitis B-related acute-on-chronic liver failure. Sci Rep 5: 13098, 2015.

10. Peng D, Wang H, Li L, Ma X, Chen Y, Zhou H, Luo Y, Xiao Y and Liu L: miR-34c-5p promotes eradication of acute myeloid leukemia stem cells by inducing senescence through selective RAB27B targeting to inhibit exosome shedding. Leukemia 32: $1180-1188,2018$

11. Re M, Magliulo G, Gioacchini FM, Bajraktari A, Bertini A, Ceka A, Rubini C, Ferrante L, Procopio AD and Olivieri F: Expression levels and clinical significance of miR-21-5p, miR-let-7a, and miR-34c-5p in laryngeal squamous cell carcinoma. Biomed Res Int 2017: 3921258, 2017.

12. Wu Z, Wu Y, Tian Y, Sun X, Liu J, Ren H, Liang C, Song L, $\mathrm{Hu} \mathrm{H}$, Wang L and Jiao B: Differential effects of miR-34c-3p and miR-34c-5p on the proliferation, apoptosis and invasion of glioma cells. Oncol Lett 6: 1447-1452, 2013.

13. Zhang JY and Xu ZS: Notch signal pathway and chronic lymphocytic leukemia. Zhongguo Shi Yan Xue Ye Xue Za Zhi 22: 1472-1475, 2014 (In Chinese).

14. Yang H, Li Y, Li T, Xu M, Chen Y, Wu C, Dang X and Liu Y: Multifunctional core/shell nanoparticles cross-linked polyetherimide-folic acid as efficient Notch-1 siRNA carrier for targeted killing of breast cancer. Sci Rep 4: 7072, 2014.

15. Lähdeniemi I, Misiorek JO, Antila CJM, Landor SKJ, Stenvall CGA, Fortelius LE, Bergström LK, Sahlgren C and Toivola DM: Keratins regulate colonic epithelial cell differentiation through the Notch1 signalling pathway. Cell Death Differ 24 984-996, 2017.

16. Sharif A, Shaji A, Chammaa M, Pawlik E and Fernandez-Valdivia R: Notch transduction in non-small cell lung cancer. Int J Mol Sci 21: 5691, 2020.

17. Sui C, Zhuang C, Sun D, Yang L, Zhang L and Song L: Notch1 regulates the JNK signaling pathway and increases apoptosis in hepatocellular carcinoma. Oncotarget 8: 45837-45847, 2017.

18. Livak KJ and Schmittgen TD: Analysis of relative gene expression data using real-time quantitative PCR and the 2(-Delta Delta C(T)) method. Methods 25: 402-408, 2001

19. Li Y, Ding Y, Ding N, Zhang H, Lu M, Cui X and Yu X: MicroRNA-625-5p sponges lncRNA MALAT1 to inhibit cervical carcinoma cell growth by suppressing NF- $\mathrm{kB}$ signaling. Cell Biochem Biophys 78: 217-225, 2020.

20. Li B, Wu N, Zhang XJ, Wei ZL and Shang LX: MicroRNA-409 inhibits the proliferative ability of cervical carcinoma cells by regulating AKT. Eur Rev Med Pharmacol Sci 22: 936-942, 2018

21. Ma C, Xu B, Husaiyin S, Wang L, Wusainahong K, Ma J, Zhu K and Niyazi M: MicroRNA-505 predicts prognosis and acts as tumor inhibitor in cervical carcinoma with inverse association with FZD4. Biomed Pharmacother 92: 586-594, 2017.
22. Wei S, Zhang ZY, Fu SL, Xie JG, Liu XS, Xu YJ, Zhao JP and Xiong WN: Hsa-miR-623 suppresses tumor progression in human lung adenocarcinoma. Cell Death Dis 8: e2829, 2017.

23. Huang F, Lin C, Shi YH and Kuerban G: MicroRNA-101 inhibits cell proliferation, invasion, and promotes apoptosis by regulating cyclooxygenase- 2 in HeLa cervical carcinoma cells. Asian Pac J Cancer Prev 14: 5915-5920, 2013.

24. Ham O, Lee CY, Kim R, Lee J, Oh S, Lee MY, Kim J, Hwang KC, Maeng LS and Chang W: Therapeutic potential of differentiated mesenchymal stem cells for treatment of osteoarthritis. Int J Mol Sci 16: 14961-14978, 2015.

25. Wu H, Huang M, Lu M, Zhu W, Shu Y, Cao P and Liu P: Regulation of microtubule-associated protein tau (MAPT) by miR-34c-5p determines the chemosensitivity of gastric cancer to paclitaxel. Cancer Chemother Pharmacol 71: 1159-1171, 2013.

26. Grisanti L, Revenkova E, Gordon RE and Iomini C: Primary cilia maintain corneal epithelial homeostasis by regulation of the notch signaling pathway. Development 143: 2160-2171, 2016.

27. Eberhart C: Multiple cilia suppress tumour formation. Nat Cell Biol 18: 368-369, 2016.

28. Giannone G, Attademo L, Scotto G, Genta S, Ghisoni E, Tuninetti V, Aglietta M, Pignata S and Valabrega G: Endometrial cancer stem cells: Role, characterization and therapeutic implications. Cancers (Basel) 11: 1820, 2019.

29. Shen M, Dong C, Ruan X, Yan W, Cao M, Pizzo D, Wu X, Yang L, Liu L, Ren X and Wang SE: Chemotherapy-induced extracellular vesicle miRNAs promote breast cancer stemness by targeting ONECUT2. Cancer Res 79: 3608-3621, 2019.

30. Wang S, Cai L, Zhang F, Shang X, Xiao R and Zhou H: Inhibition of EZH2 attenuates sorafenib resistance by targeting NOTCH1 activation-dependent liver cancer stem cells via NOTCH1-related MicroRNAs in hepatocellular carcinoma. Transl Oncol 13: 100741,2020

31. Lu C, Ren C, Yang T, Sun Y, Qiao P, Wang D, Lv S and Yu Z: A noncanonical role of fructose-1, 6-bisphosphatase 1 is essential for inhibition of Notch1 in breast cancer. Mol Cancer Res 18: 787-796, 2020.

32. Yang LZ, Lei CC, Zhao YP, Sun HW, Yu QH, Yang EJ and Zhan X: MicroRNA-34c-3p target inhibiting NOTCH1 suppresses chemosensitivity and metastasis of non-small cell lung cancer. J Int Med Res 48: 300060520904847, 2020.

33. Hu J, Yu J, Gan J, Song N, Shi L, Liu J, Zhang Z and Du J: Notch $1 / 2 / 3 / 4$ are prognostic biomarker and correlated with immune infiltrates in gastric cancer. Aging (Albany NY) 12: 2595-2609, 2020.

(i) $\Theta$ This work is licensed under a Creative Commons Attribution-NonCommercial-NoDerivatives 4.0 International (CC BY-NC-ND 4.0) License. 